\title{
Zwei Drittel der Demenzkranken erhalten potenziell inadäquate Medikamente
}

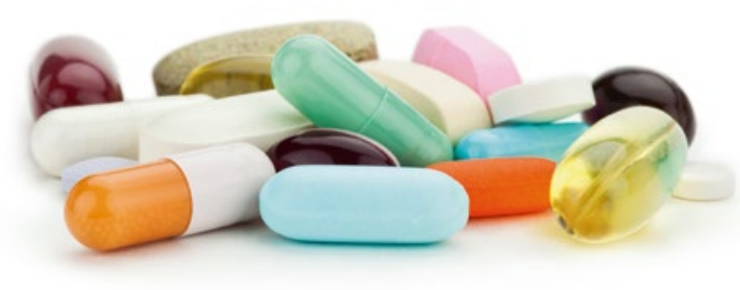

Ältere Demenzkranke werden häufig mit potenziell inadäquaten Medikamenten (PIM) behandelt. Zwei Substanzgruppen stehen dabei im Vordergrund.

$\mathrm{F}$ ast zwei Drittel aller Demenzpatienten erhalten mindestens ein PIM, und über ein Viertel sogar zwei oder mehr. Das hat die europäische Studie „RightTimePlaceCare“ (RTPC) zutage gefördert, in der bei 2.004 älteren Menschen mit Demenz die Eignung der Medikation anhand der EU(7)-PIM-Liste überprüft wurde.

Bei den einzelnen PIM wird die Rangliste angeführt von Protonenpumpeninhibitoren (PPI) für mehr als sechs Wochen, gefolgt von Risperidon für mehr als acht Wochen. Aufgeschlüsselt nach Substanzgruppen ergibt sich die umgekehrte Reihenfolge: Hier stehen Neuroleptika auf Platz eins, sie machen $26 \%$ al- ler PIM-Verordnungen aus. Auf „Medikamente für säureassoziierte Erkrankungen" entfallen $21 \%$.

Das Risiko, ein PIM rezeptiert zu bekommen, ist der Studie zufolge besonders hoch, wenn die Patienten schon 80 oder älter sind, in einem Pflegeheim leben, viele Begleiterkrankungen haben und/oder funktionell eingeschränkt sind. Mit fortschreitenden Kognitionseinbußen geht die PIM-Rate dagegen zurück. Ob zurückhaltender verordnet wird oder Erkrankungen seltener erkannt werden, lässt sich aus der Studie nicht ableiten.

PIM sollten generell bei alten Menschen möglichst vermieden werden, weil das Risiko für unerwünschte Wirkun- gen den klinischen Nutzen übersteigt und oft alternative Medikamente mit größerer Sicherheit und Effektivität verfügbar sind. Der Einsatz von Neuroleptika ist darüber hinaus speziell bei Demenzpatienten mit einem höheren Sterberisiko verbunden.

\section{PIM erhöhen die Sturzneigung}

Die RTPC-Studie liefert auch Hinweise auf mögliche schädliche Auswirkungen der PIM-Gabe: Die Risiken, innerhalb von drei Monaten eine sturzbedingte Verletzung zu erleiden bzw. in ein Krankenhaus aufgenommen zu werden, sind demnach mit PIM gut um die Hälfte höher als ohne.

Renom-Guiteras A et al. Age and Ageing 2017, online 1. September 2017;

https://doi.org/10.1093/ageing/afx147

\section{Doppelt geschützt}

\section{Weniger funktionelle Beeinträchtigungen bei herzgesunden Senioren}

\section{Die Förderung der kardiovaskulären Gesundheit ist nicht nur für die Vermei- dung von Gefäßkomplikationen relevant, sie kann auch den funktionellen Abbau bremsen.}

\footnotetext{
$\bullet$ ber den Zusammenhang zwischen kardiovaskulärer Gesundheit und funktionellen Fähigkeiten berichten Ärzte aus Chile, die repräsentativ ausgewählte und nicht in einem Heim lebende über 65-Jährige befragt haben. Von 460 Teilnehmern im mittleren Alter von 73 Jahren, davon knapp 60\% Frauen, gaben rund $42 \%$ an, erhebliche Schmerzen und/oder gravierende Schwierigkeiten bei Mobilität, Selbstversorgung, Kognition, sozialem Austausch, Visus, Schlaf oder Stimmung zu haben.

Das Risiko, von mindestens einer dieser Einschränkungen betroffen zu sein, war bei einer idealen kardiovaskulären
}

Gesundheit um 49\% und bei einer mittleren um 36\% geringer als bei einer schlechten. Die Risikoreduktionen waren unabhängig von Unterschieden in Alter und medizinischer Vorgeschichte der Senioren, inklusive Stürzen, Arthrose und Gefäßerkrankungen.

Eine ideale kardiovaskuläre Gesundheit bestand per Definition, wenn von vier Lebensstilfaktoren und drei physiologischen Parametern mindestens fünf erfüllt waren. Die Lebensstilkriterien waren wie folgt definiert: $\mathrm{BMI}<25 \mathrm{~kg} / \mathrm{m}^{2}$, körperliche Aktivität $\geq 600$ metabolische Äquivalente (MET) pro Woche, Tabakabstinenz und gesun- de Ernährung. Die physiologischen Kriterien waren: Blutdruck $<120 / 80 \mathrm{mmHg}$, Gesamtcholesterinwert $\leq 200 \mathrm{mg} / \mathrm{dl}$ und Nüchtern-Blutzuckerspiegel $<100$ $\mathrm{mg} / \mathrm{dl}$, jeweils ohne Behandlung. Wurden maximal zwei Kriterien erreicht, entsprach dies einer schlechten kardiovaskulären Gesundheit.

\section{Herzgesunde waren mobiler und selbstständiger}

Eine gute kardiovaskuläre Gesundheit wirkte sich vor allem im Hinblick auf die Mobilität und die Fähigkeit zur Selbstversorgung im Alter positiv aus. Bei idealem bzw. intermediärem Abschneiden war das Risiko für gravierende Beeinträchtigungen in diesen Bereichen um $77 \%$ und $75 \%$ bzw. um 39\% und 38\% reduziert. V.a. eine ideale körperliche Aktivität und ein idealer BMI waren mit einem geringeren Ausmaß an Einschränkungen verknüpft.

bs

García-Hermoso A et al. J Am Geriatr Soc 2017, online 25. Oktober 2017;

https://dx.doi.org/10.1111/jgs.15139 